\title{
EL SOLUTRENSE MERIDIONAL IBÉRICO: EL NÚCLEO DE LA PROVINCIA DE MÁLAGA
}

\section{THE SOUTHERN IBERIAN SOLUTREAN: THE CORE OF THE PROVINCE OF MÁLAGA}

\author{
MARÍA DOLORES SIMÓN VALLEJO* \\ MARÍA MERCÈ BERGADÀ ZAPATA** \\ JUAN FRANCISCO GIBAJA BAO*** \\ MIGUEL CORTÉS SÁNCHEZ****
}

\begin{abstract}
Resumen: La provincia de Málaga es una de las áreas con mayor número de yacimientos solutrenses del sur de la Península Ibérica. En este trabajo abordamos el contexto estratigráfico, sedimentario y paleoambiental del Solutrense en esta área, sobre todo a partir de los análisis efectuados en Cueva Bajondillo, y tratamos su correlación con otros registros del Mediterráneo ibérico.

Así mismo, el análisis de la secuencia tecnocultural, las estrategias de subsistencia desde una perspectiva morfológica y funcional o de las manifestaciones simbólicas nos permiten identificar los principales rasgos culturales del Solutrense en este ámbito geográfico.
\end{abstract}

Palabras clave: Málaga, Solutrense, Paleoambiente, Secuencia, Simbolismo.

\section{LOS YACIMIENTOS SOLUTRENSES DE MÁLAGA: MARCO HISTORIOGRÁFICO}

La provincia de Málaga reúne una de las mayores concentraciones conocidas de yacimientos arqueológicos del Pleistoceno superior del sur de la Península Ibérica (Cortés y Sanchidrián 1999). No obstante, la

\footnotetext{
* Museo Arqueológico de Frigiliana, c/ Cuesta del Apero, 10 29788-Frigiliana, Málaga.mm.cosi@terra.es

** SERP. Departamento de Prehistoria, Historia Antigua y Arqueología. Facultad de Geografía e Historia. Universidad de Barcelona, c/ Montealegre, 6-8.08001-Barcelona.bergada@ub.edu
}

\begin{abstract}
The province of Malaga is one of the areas with the greatest number of Solutrean sites in southern Iberia. In this paper we treat the sedimentary, chronological and paleoenvironmental contexts of the Solutrean in this area, especially from the analysis in Bajondillo Cave. We also analysed its correlation with other records in the Iberian Mediterranean.

Also, the techno-sequence analysis, the subsistence strategies as well as the symbolic manifestations allow the identification of the main characteristics of the regional Solutrean.
\end{abstract}

Key words: Málaga, Solutrean, Paleoenvironment, Sequence, Simbolism.

distribución de los emplazamientos sobre esta circunscripción administrativa de $7.306 \mathrm{~km}^{2}$ es desigual pues la mayoría de ellos aparecen concentrados en las proximidades de la costa actual y sólo unos pocos se localizan en el interior (Fig. 1, Tabla 1). A esta repartición heterogénea hay que añadir que los enclaves localizados en el área de influencia costera destacan también porque son

*** Departamento de Arqueología CSIC-IMF. Investigador Ramón y Cajal, c/ Egipciaques, 15. 08001-Barcelona. jfgibaja@imf.csic.es

**** Departamento de Prehistoria y Arqueología. Facultad de Geografía e Historia, Universidad de Sevilla, c/ María de Padilla s/n. 41004-Sevilla.mcortes@us.es 


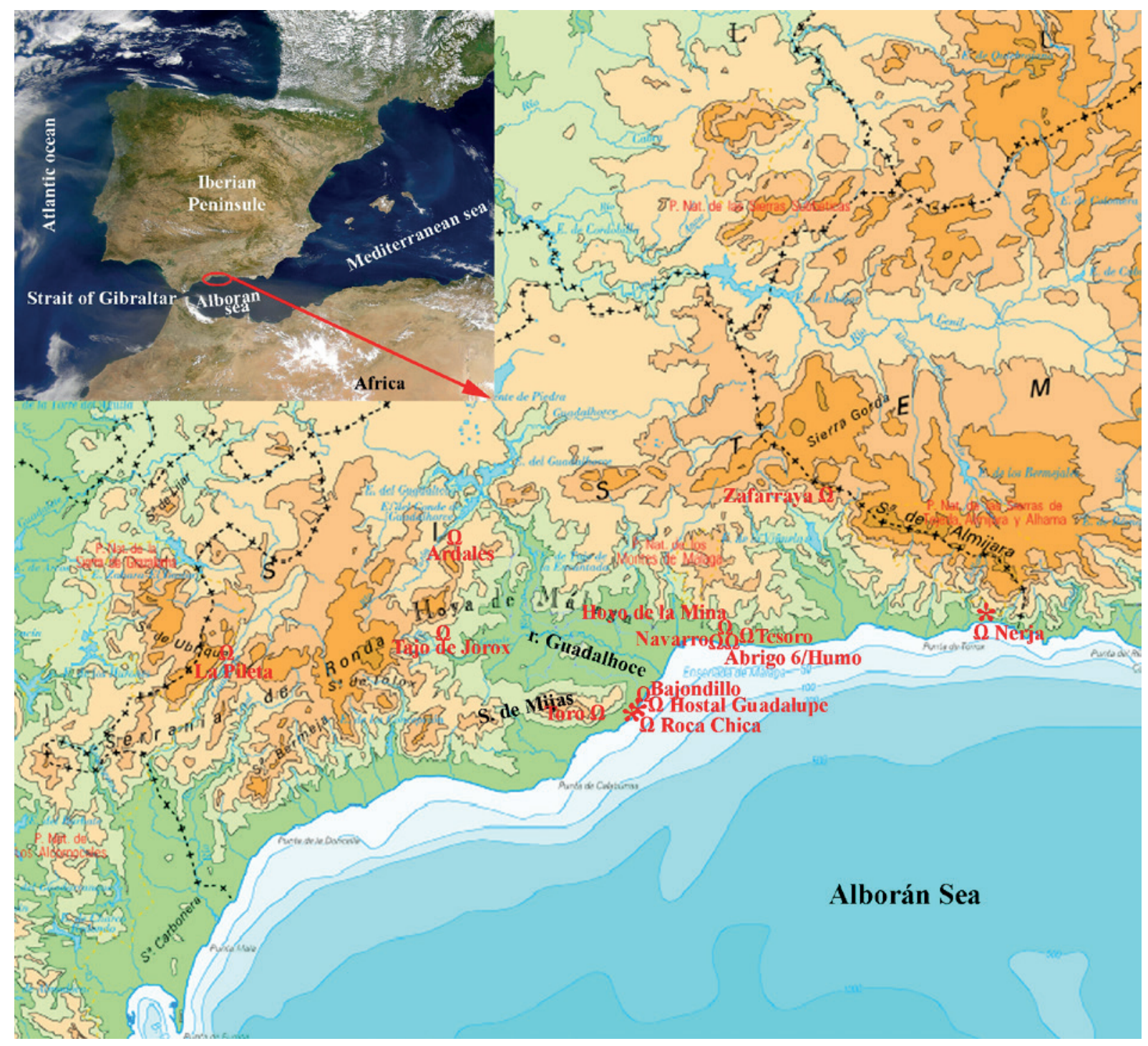

Figura1. Mapa de la provincia de Málaga con la ubicación de los yacimientos citados en el texto.

los que disponen de unas secuencias estratigráficas mejor contrastadas. Así, de occidente a oriente tendríamos las siguientes cavidades (Tabla 1, Figura 1):

Cueva del Toro o Calamorro (Benalmádena). Se trata de un yacimiento con arte parietal (Fortea y Giménez 1973). Hasta el momento no se ha realizado ningún sondeo, aunque es previsible conserve algún relleno pues en superficie se documentan materiales neolíticos (Cortés et al. 2010).

Cueva Bajondillo, en realidad un gran abrigo abierto en el edificio travertínico de Torremolinos, cuenta con 19 estratos arqueológicos, de los cuales cuatro de ellos $(\mathrm{Bj} / 9 \mathrm{a} \mathrm{Bj} / 6)$ han sido atribuidos al Solutrense pleno
(Bj/9) y evolucionado (Bj/8 a 6) (Cortés 2007b). De este segmento de la secuencia disponemos de registros polínicos, isotópicos, sedimentológicos, microestratigráficos y cronológicos. En este último apartado, cuatro dataciones, una de ${ }^{14} \mathrm{C}$-AMS y tres de termoluminiscencia (Tabla 2), permiten contextualizar cronológicamente este tramo estratigráfico.

Cueva Navarro IV (Málaga) es un yacimiento de arte parietal caracterizado por la existencia de un único zoomorfo (un uro) junto con numerosos signos simples atribuidos al Solutrense (Sanchidrián 1981). La existencia de diversos artefactos líticos tallados en superficie recogidos por este autor y el hecho de que la boca 
Tabla 1. Yacimientos de la provincia de Málaga

\begin{tabular}{|c|c|c|c|c|c|c|}
\hline $\begin{array}{l}\text { Yacimiento } \\
\text { en cueva }\end{array}$ & Municipio & $\begin{array}{c}\text { Ámbito } \\
\text { geográfico }\end{array}$ & m. s.n.m. & $\begin{array}{c}\text { Secuencia } \\
\text { estratigráfica }\end{array}$ & Arte & Fuente \\
\hline Toro & Benalmádena & \multirow{10}{*}{ Costa } & 500 & Sin sondear & Parietal & Fortea y Giménez 1973 \\
\hline Roca Chica & Torremolinos & & 5 & Sin sondear & No & Inédito \\
\hline Bajondillo & Torremolinos & & 15 & $\mathrm{Bj} / 6-\mathrm{Bj} / 7-\mathrm{Bj} / 8-\mathrm{Bj} / 9$ & Mobiliar & Cortés 2007a \\
\hline Hoyo de la Mina & Málaga & & 125 & $\mathrm{HM} / 7$ & No & Ferrer et al. 2006 \\
\hline Navarro & Málaga & & c. $10^{*}$ & Sin sondear & Sí & Sanchidrián 1981 \\
\hline $\begin{array}{c}\text { Abrigo 4 / } \\
\text { Complejo Humo }\end{array}$ & Málaga & & 5 & Tramo medio & No & Ramos y Durán 1998 \\
\hline $\begin{array}{c}\text { Abrigo 6/ } \\
\text { Complejo Humo }\end{array}$ & Málaga & & 16 & Estrato 10 & No & Ramos et al. 2006 \\
\hline Victoria & $\begin{array}{l}\text { Rincón de la } \\
\text { Victoria }\end{array}$ & & 70 & Sin sondear & Sí & Cantalejo et al., 2007 \\
\hline Higuerón & $\begin{array}{l}\text { Rincón de la } \\
\text { Victoria }\end{array}$ & & 80 & No & Sí & $\begin{array}{l}\text { Cacho y López } 1979 \\
\text { Cantalejo et al. } 2007\end{array}$ \\
\hline Nerja & Nerja & & 158 & $\begin{array}{c}\text { V/IX-VIII-VII } \\
\text { (Quadra 1962.1963) } \\
\text { V/9-8-7 } \\
\text { (Jordá 1979-187) } \\
\text { Mina-80A/8-7 } \\
\text { (Pellicer 1980) }\end{array}$ & Sí & $\begin{array}{c}\text { Cortés et al., } 2006 \\
\text { Simón 2003 } \\
\text { Aura et al. 2001, 2006, } \\
\text { Jordá } 1986 \\
\text { Jordá y Aura } 2006 \\
\text { Cava } 1997\end{array}$ \\
\hline La Pileta & Benaoján & \multirow{4}{*}{ Interior } & 670 & Sí & Sí & $\begin{array}{l}\text { Breuil et al. } 1915 \\
\text { Cortés y Simón } 2008\end{array}$ \\
\hline Cueva de Ardales & Ardales & & 565 & Material superficie & Sí & Cantalejo et al. 2006 \\
\hline Tajo de Jorox & Alozaina & & 570 & $\begin{array}{l}\text { Colección } \\
\text { sin contexto }\end{array}$ & Sí & Marqués y Ruiz 1976 \\
\hline Zafarraya & Alcaucín & & 1.100 & Sí & No & Barroso 2006 \\
\hline
\end{tabular}

* Se trata de un valor estimativo pues la entrada original se encuentra obstruida por derrubios y el acceso se realiza desde la parte final de la cueva.

Tabla 2. Dataciones efectuadas en los yacimientos de Bajondillo y Nerja

\begin{tabular}{|c|c|c|c|c|c|}
\hline Estrato & AMS & Cal. BP ** & TL & Muestra & Laboratorio \\
\hline Bajondillo/6 & - & - & - & - & - \\
\hline Bajondillo/7 & - & - & $16.438 \pm 1497$ & Carbonatos & MAD-3927 \\
\hline Nerja V8/s* & $17.940 \pm 200$ & $19638 \pm 459$ & - & Hogar & UBAR-98 \\
\hline Bajondillo/8 & - & - & $17.582 \pm 1521$ & Carbonatos & MAD-3926 \\
\hline Nerja V/8k-1* & $18.420 \pm 530$ & $20104 \pm 663$ & - & Carbón & UBAR-158 \\
\hline Bajondillo/9 & - & - & $18.701 \pm 2154$ & Sílex & MAD-2405 \\
\hline Bajondillo/9 & $19.990 \pm 480$ & $23886 \pm 596$ & - & Hueso & AA 34710 \\
\hline Nerja-V/9a* & $21.140 \pm 190$ & $23346 \pm 397$ & - & Bráctea Pinus sp. & - \\
\hline
\end{tabular}

*Aura et al. 2006, **CalPal2007_HULU 
de entrada a la cavidad se encuentre sellada hace mantener expectativas sobre la presencia de niveles ocupacionales paleolíticos.

Cueva del Hoyo de la Mina (Málaga) es una cavidad conocida a partir de las excavaciones de M. Such (1920) y, sobre todo, por el análisis posterior de J. Fortea (1973). Recientes trabajos han permitido constatar la secuencia estratigráfica del yacimiento, en la que se documenta la presencia de arpones en la serie magdaleniense $\mathrm{y}$, como novedad más significativa para este trabajo, la presencia de niveles solutrenses que han aportado un utillaje diagnóstico muy claro que permite ser atribuido al Solutrense evolucionado, no final (Ferrer et al. 2006).

El Complejo kárstico de Humo (Málaga) dispone de varios abrigos y cuevas, de los cuales los abrigos 4 y 6 han aportado indicios atribuibles al Solutrense. El Abrigo 4 consta de una amplia secuencia estratigráfica de $14 \mathrm{~m}$ de potencia, no sondeada hasta el momento de forma sistemática, y de la cual procede una colección industrial que permite identificar la presencia de ocupaciones solutrenses (Ramos et al. 2006). Abrigo 6 es el yacimiento que podría está llamado a ser la referencia para el sector oriental de la bahía de Málaga pues los sondeos practicados hasta el momento, de poca envergadura, han entregado un rico registro atribuible a la Prehistoria reciente, Epipaleolítico, Magdaleniense y Solutrense, en este caso parece que pleno (Ramos et al. 2006).

Cueva Victoria (Rincón de la Victoria) es otra de las cavidades que conserva arte parietal atribuible al Solutrense, con un único uro y diversos signos asociados (Cantalejo et al. 2007), si bien el registro estratigráfico, apenas sondeado por el momento, se limita a niveles neolíticos, epipaleolíticos y magdalenienses (Fortea 1973).

Cueva del Higuerón (Rincón de la Victoria) es conocida dentro de la historiografía solutrense por algunos artefactos diagnósticos recuperados en actividades no arqueológicas (Cacho y López 1979). No obstante, el vaciado integral e incontrolado de la caverna ha hecho desaparecer posiblemente uno de los mejores registros de la costa de Málaga. Así mismo, la cavidad dispone de diversos vestigios gráficos adscribibles al Solutrense.

Cueva de Nerja (Nerja) es el yacimiento con la secuencia del Pleistoceno superior reciente mejor contrastada hasta el momento en la costa de Málaga y, sin duda, una de las referencias claves para el conocimiento de los aspectos secuenciales, económicos o simbólicos del Paleolítico superior del sur de Iberia (vid. p.ej. Jordá 1986, Jordá y Aura 2006, Aura et al. 2001, 2002, 2006, Simón 2003). La secuencia solutrense, sistematizada recientemente (Aura et al. 2006), se desglosa en una fase A, la más antigua y que correría paralela al desarrollo del Solutrense inferior ibérico; otra plena o B, que sería sincrónica al Solutrense medio/pleno ibérico; y por último la $\mathrm{C}$, más reciente, que sería coincidente con el Solutrense evolucionado de facies ibérica. Las dataciones permiten ajustar relativamente la cronología del Solutrense en el yacimiento (Tabla 2).

Además de estos enclaves hay otros emplazamientos con algunos indicios pendientes de contrastación, como sería el caso de Cueva de la Roca Chica (Torremolinos). Aunque esta cavidad, localizada en las inmediaciones de la costa, fue parcialmente destruida en la década de los años setenta del siglo XX se conservan diversos materiales depositados en la Sección de Arqueología del Museo Provincial de Málaga que apuntan hacia la existencia de industrias atribuibles al Paleolítico superior (Cortés 2010). Así mismo, la ausencia de sondeos estratigráficos tanto en Toro como en Navarro o la falta de profundización en la secuencia de Victoria hacen mantener expectativas sobre la posibilidad de que estas cavidades dispongan de depósitos potenciales para ampliar el conocimiento del Solutrense en la provincia de Málaga.

Por su parte, los yacimientos del hinterland (Fig. 1, Tabla 1) aportan una información secuencial más pobre pero significativamente más rica en el apartado simbólico.

Cueva de La Pileta (Benaoján) fue el primer yacimiento del sur peninsular cuyas manifestaciones artísticas parietales fueron atribuidas al Solutrense. Además, la cavidad cuenta con la más rica y compleja disposición de pinturas y grabados de toda la mitad meridional de Iberia (Breuil et al. 1915, Sanchidrián 1997). Así mismo, la revisión de los materiales arqueológicos recuperados en la intervención de 1943 en la Sala de los Murciélagos permite apuntar la presencia de una secuencia arqueológica muy amplia, en la que probablemente estén representadas ocupaciones solutrenses (Cortés y Simón 2008).

Cueva de Ardales o de Doña Trinidad (Ardales) constituye un yacimiento de primer orden pues muestra una secuencia gráfica que arrancaría en el Paleolítico superior inicial (Gravetiense) y cubriría el Solutrense y el Magdaleniense con una importantísima colección de motivos reconocidos a partir de una exhaustiva labor de investigación (Breuil 1921, Cantalejo et al. 2006). Así mismo, recientemente se ha puesto en marcha un ambicioso proyecto general de investigación sobre el relleno sedimentario de la cavidad que, a buen seguro, deparará importantes novedades a corto plazo. 
Finalmente, se han definido como solutrenses algunas piezas líticas descontextualizadas recogidas en Tajo de Jorox (Alozaina) (Marqués y Ruiz 1976), Ardales (Cantalejo et al. 2006), una colección de materiales procedente de los niveles superiores de relleno de Cueva del Boquete de Zafarraya (Alcaucín) (Barroso et al. 2006) y las ya mencionadas de La Pileta (Cortés y Simón 2008).

La secuencia de todos los yacimientos solutrenses con estratigrafía (Nerja, Abrigo 4 y 6 del Complejo del Humo, Zafarraya, Bajondillo y Hoyo de la Mina) y los demás indicios expuestos definen un conjunto de caracteres que entroncan con el esquema general del Solutrense Ibérico (Cortés 2007b) tanto desde el punto de vista tecnológico como tipológico o, en cuando existen dataciones, cronológico.

No obstante, los datos procedentes de los yacimientos mejor conocidos, Nerja (Aura et al. 2006) y Bajondillo (Cortés 2007a), son aún poco resolutivos para articular una propuesta de evolución detallada como la existente para el sector central del Mediterráneo Ibérico. Así, los datos sobre los momentos más antiguos del Solutrense son muy pobres, de modo que sólo puede apuntarse que Nerja y Bajondillo parecen mostrar entre ca. 21-19ka B.P. unos conjuntos industriales marcados todavía por una fuerte influencia gravetiense a la que se incorporan algunos elementos tipológicos solutrenses. Si esta tendencia se confirmara en nuevos trabajos y registros quizás podría concretarse un proceso de atomización geográfica del Gravetiense final de facies ibérica previa a la generalización de los procesos tecnológicos solutrenses (Cortés 2007b) que, en la actualidad, constituye más una hipótesis que un hecho contrastado.

El resto de niveles, colecciones y yacimientos pueden atribuirse por la presencia/ausencia de elementos diagnósticos (empleando para ello la seriación levantina para el Solutrense de facies ibérica), y en algún caso de dataciones disponibles, a un Solutrense medio-evolucionado. Así, las fases más antiguas quedarían asociadas a la presencia de piezas de cara plana y algún foliáceo (Nerja Vestíbulo/8 y Bj/9), en tanto que el Solutrense superior y evolucionado estaría caracterizado por la presencia de puntas de aletas y pedúnculo y de escotadura/muesca (Bajondillo/6-8, Nerja/ Mina-80A-8, 7 y Vestíbulo-7, Higuerón, Tajo de Jorox, Abrigo 6 y Zafarraya).

En el caso de La Pileta, el utillaje solutrense se limita a una punta de cara plana pero el aspecto general y de conjunto del utillaje complementario (raspadores, buriles, etc.) podría quizás sintonizar mejor con momentos plenos del Solutrense (Cortés y Simón 2008).
En otro orden de cosas, las colecciones procedentes de estos yacimientos, como ocurre en muchos de los emplazamientos de la mitad meridional ibérica, aparecen caracterizadas por una notable presencia de buriles (Cortés y Simón 1997).

\section{SECUENCIA ESTRATIGRÁFICA Y APROXIMACIÓN PALEOAMBIENTAL}

A la hora de establecer la secuencia estratigráfica, sedimentaria y paleoambiental solutrense en el sur peninsular nos hemos centrado principalmente en el yacimiento de Bajondillo (Bergadà y Cortés 2007, Cortés et al. 2008, 2011), aunque también hemos establecido correlaciones con el registro de Cueva de Nerja (Jordá 1986, Jordá y Aura 2006, Aura et al. 2002, Aura et al. 2006, Aura et al. 2010).

Para relacionar los episodios documentados con la cronoestratigrafía basada en los proxies de alta resolución, obtenidos a partir del estudio de los testigos de hielo de los sondeos de Groenlandia, especialmente del GRIP y del NGRIP (Lowe et al. 2008), se han calibrado las fechas radiocarbónicas mediante la curva de calibración CalPal2007-Hulu (Weninger et al. 2004). Finalmente, hemos comparado las curvas de probabilidad acumulada de las fechas calibradas con la del GIC005 (Lowe et al. 2008) y también se ha tenido en cuenta la curva de variación de la temperatura de la superficie del mar, construida a partir del análisis de las alquenonas del sondeo MD95-2043 situado en el Mar de Alborán en el sur de Málaga (Cacho et al. 2001).

El registro que proporciona Bajondillo (Fig. 2) muestra la existencia de un hiatus entre el horizonte con industrias gravetienses $(\mathrm{Bj} / 10)$ y las primeras ocupaciones solutrenses $(\mathrm{Bj} / 9)$. Por su parte, en Nerja se detecta un proceso erosivo neto entre las unidades 1 (niveles gravetienses) y 2 (ocupaciones solutrenses).

El primer episodio sedimentario adscrito al Solutrense de Bajondillo está fechado entre $c a .19^{\prime} 9 \mathrm{ka}$ B.P. (AMS de Bj/9) - 17'5ka B.P. (TL de Bj/8) representado por Bajondillo $T(\mathrm{Bj} / 9$, Solutrense pleno y $\mathrm{Bj} / 8$, Solutrense evolucionado) y caracterizado, en un inicio, por aportes eólicos vinculados a un ambiente árido y frío, muy especialmente en $\mathrm{Bj} / 9$, mientras que, en una fase posterior, se acentúa un proceso de gelifluxión, caracterizando un ambiente más húmedo. Esta fase se situaría con la máxima probabilidad (95\%) entre 24970 y 22930 años cal BP (Fig. 3) y se adscribiría bien al final del GS-3, en las postrimerías del evento frío Heinrich 2, bien a los inicios del GS-2c (Cortés 


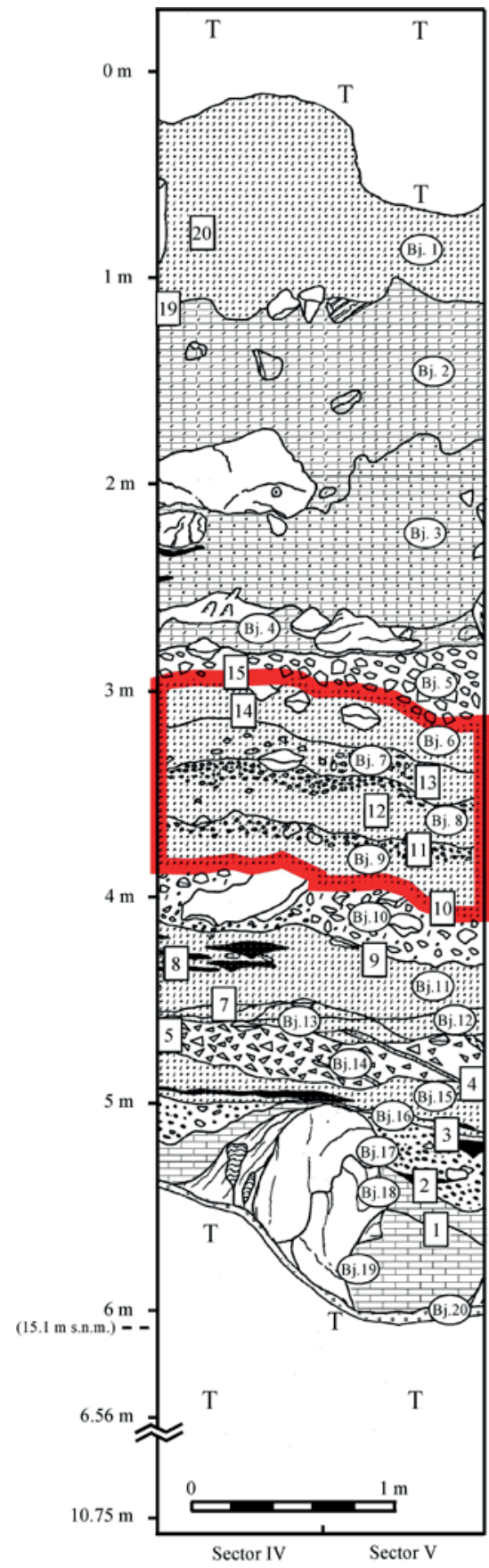

\section{Litoestratigrafía de \\ Cueva de Bajondillo \\ Perfil Oeste}

(Tramo central. Sector IV-V)

\section{Horizontes}

Solutrenses

\begin{tabular}{|c|c|}
\hline $\mathrm{T}$ & Travertino de pared y techo \\
\hline 臣罣 & Travertino \\
\hline 匿 & Travertino arenoso \\
\hline 㞔目 & Travertino limoso \\
\hline 唡 & Arenas limosas carbonatadas \\
\hline 再雨 & Limos arenosos carbonatados \\
\hline 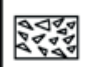 & Cantos angulosos de travertino \\
\hline 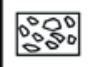 & Cantos subangulosos \\
\hline i & Costra carbonatada \\
\hline$\overparen{6 A^{2}}$ & Espeleotema \\
\hline$E$ & Carbones y cenizas \\
\hline 1 & Muestreo micromorfológico \\
\hline
\end{tabular}

Figura 2. Litoestratigrafía de Cueva Bajondillo (Torremolinos, Málaga): registro Solutrense.

et al. 2011). Dichas condiciones fueron disminuyendo paulatinamente en $\mathrm{Bj} / 8$, fechado en $17582 \pm 1521$ (TL). Por su parte en Nerja, en la zona del Vestíbulo, aparece representado por un episodio sedimentario enmarcado por los niveles NV/10 (Solutrense A, sincrónico Solutrense al inferior de facies ibérica) y NV/9 (Solutrense asimilable por cronología al Solutrense pleno), datado entre 25770-24650 años cal BP, que presentan “unas características sedimentológicas indicativas de un clima fresco, pero más húmedo que el reconocido para la Unidad 1" (Aura et al. 2006: 71) y situados en el episodio del GS-3 (Aura et al. 2010).

El segundo episodio de Bajondillo, entre $c a$. 17'5ka (TL de $\mathrm{Bj} / 8$ ) y $<16^{\prime} 4 \mathrm{ka}(\mathrm{TL}$ de $\mathrm{Bj} / 7)$, corresponde a 


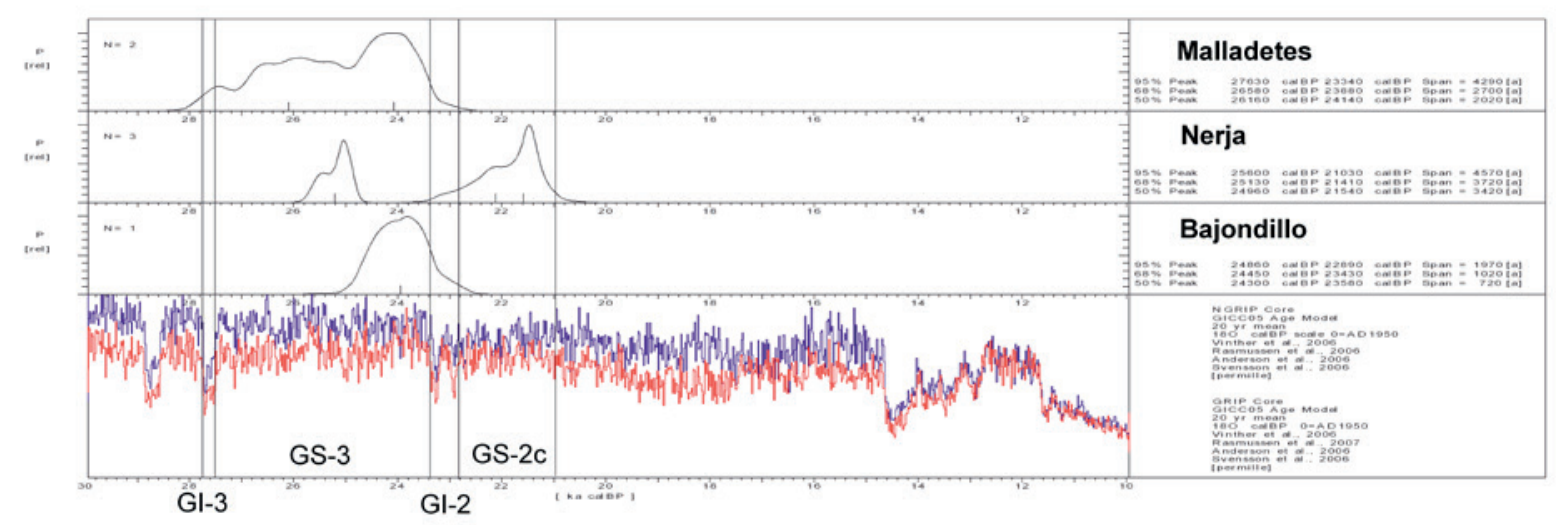

Figura 3. Curvas de probabilidad acumulada obtenidas a partir de la calibración CalPal2007- Hulu (Weninger et al. 2007) de las fechas de los yacimientos de Bajondillo, Nerja y Malladetes y su comparación con las curvas de variación de $\delta^{18} \mathrm{O}$ obtenidos de los sondeos de NGRIP y GRIP según GICC05 Age Model (Lowe et al. 2008).

Bajondillo $U(\mathrm{Bj} / 7$ y Bj/6, Solutrense evolucionado). $\mathrm{Su}$ formación responde a una disgregación de la pared con alguna caída de bloques y a un proceso de arroyada que removiliza el relleno. Tras la formación de $\mathrm{Bj} / 7$, fechado en $16438 \pm 1497$ (TL), se registraría un proceso erosivo que conllevaría la reactivación kárstica y, más tarde, la formación de espeleotemas. Con posterioridad, reaparece la sedimentación de $\mathrm{Bj} / 6$ con la misma dinámica que seguía $\mathrm{Bj} / 7$, si bien las condiciones tienden a suavizarse respecto al episodio anterior, y que podría correlacionarse con el GS-2b. En Nerja se detecta asimismo un contacto erosivo que se correlacionaría con el GI-2, con un nuevo episodio sedimentario, NV/8, con ocupaciones adscritas al Solutrense C (equiparable al Solutrense evolucionado) y que, por "sus rasgos sedimentarios indican un clima fresco $y$ húmedo que hacia el techo se hace más seco" (Aura et al. 2006: 71) y fechados entre 23380 y 20860 años cal BP (NV/8) y 22190 y 20990 años cal BP (NV 8/s) respectivamente, puede ser adscrito al GS-2c (Aura et al. 2010).

\section{LAS ESTRATEGIAS DE SUBSISTENCIA: EL APORTE DE LOS ANÁLISIS ARQUEOFAUNÍSTICOS Y TRACEOLÓGICOS}

Los estudios de fauna durante el Solutrense en la provincia de Málaga cuentan con los datos procedentes de Nerja, Abrigo 6 y Hoyo de la Mina. Dado que la información de los dos últimos permanece inédita, en la actualidad sólo disponemos de los datos procedentes de Nerja con una colección que supera los 28.000 restos (vid. Riquelme et al. 2005, Cortés et al. 2008).
Las actividades cinegéticas se concentran en la caza del conejo y la cabra montés, más esporádica sobre ciervo y jabalí y, más puntual aún, del caballo y el uro, así como de algunos pequeños carnívoros (Riquelme et al. 2005).

En relación al Gravetiense regional, la economía solutrense documentada en la costa de Málaga manifiesta la explotación de recursos marinos (p.ej. mamíferos como la foca monje, aves, peces o moluscos) desde momentos avanzados del último máximo glacial (Cortés et al. 2006). Un hecho a destacar es la presencia de fauna de carácter frío como el molusco Modiolus modiolus o las diversas aves invernantes registradas en Nerja, de clara adscripción circumboreal. Así, en el nivel Nerja/ XI y X de las excavaciones de A.M. de la Quadra, todas las especies de aves son invernantes, en el IX suponen el $55 \%$ y en el VIII representan el $70 \%$, datos que definen un contexto paleoambiental que concuerda bien con condiciones propias del Último Máximo Glacial (Cortés et al. 2006).

Los recursos subsistenciales explotados por la paleocomunidades solutrenses eran ciertamente diversificados y basados en una depredación sistemática de mamíferos de pequeño (conejo) y mediano tamaño (cabra y ciervo), aves marinas acuáticas y no acuáticas, peces y moluscos marinos y continentales (Cortés et al. 2006).

Por su parte, el estudio traceológico realizado sobre el utillaje de Nerja nos ha permitido constatar que, aunque existe una amplia variedad de materias trabajadas con los instrumentos líticos, sobresale especialmente el uso destinado al descarnado de animales, el tratamiento 


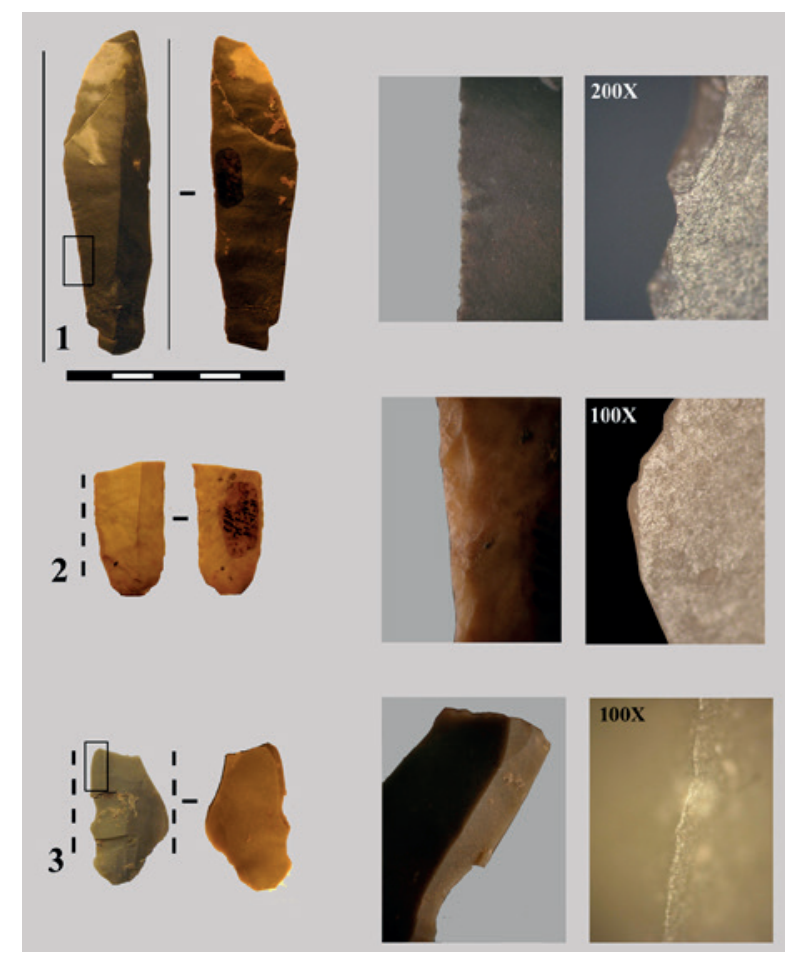

Figura 4. Instrumentos usados para: 1. Descarnar, 2. Raspado de piel seca y 3. Raspado de hueso con buril. Fotos macro a $40 \mathrm{X}$ y micro a $200 \mathrm{X}$.

de la piel y el trabajo del hueso. Asimismo y aunque de manera testimonial, hemos registrado algún posible proyectil y unas pocas piezas empleadas sobre madera, piedra y vegetal indeterminado (Fig. 4).

Hay que reseñar así mismo una cierta vinculación entre determinadas características de estos útiles y el trabajo realizado. Así, para el corte de materias blandas como la carne y la piel se han seleccionado soportes laminares de filos muy agudos sin retocar. En cambio para las tareas de raspado, caso de la piel, el hueso o la madera se eligieron lascas o láminas de filos más abruptos, habitualmente retocados. Entre estas últimas hemos documentado algunas con retoque lateral, buriles, muescas o raspadores. En este punto nos parece interesante destacar el hecho de que haya instrumentos empleados sobre piel seca y fresca, ya que ello nos indica que quizás todo el abanico de tareas necesarias en el tratamiento de esta materia se hacía en el propio asentamiento.

En cuanto al resto de útiles, cabe apuntar el empleo de una laminilla y una laminilla de dorso como posibles elementos de proyectil, el uso de una lasca empleada seguramente para raspar una materia mineral y la utilización de un buril para raspar una materia vegetal, quizás madera.

\section{LA ESFERA SIMBÓLICA}

El Solutrense supone con diferencia el segmento cronocultural con mayor cantidad y variedad de manifestaciones simbólicas en el área de estudio de este trabajo. Así, contamos con siete cavidades con arte rupestre atribuido a estos momentos: La Pileta, Ardales, Toro/Calamorro, Navarro, Victoria, Higuerón y Nerja (Breuil et al. 1915, Fortea y Giménez 1973, Sanchidrián 1981, 1994, 1997, Cantalejo et al. 2006, 2007).

Desde un punto de vista cuantitativo y de complejidad de los desarrollos topoiconográficos destacan La Pileta, Ardales y Nerja. En el caso de Higuerón, los vestigios gráficos se restringen a áreas recónditas que han quedado sustraídas de la degradación antrópica y natural de las paredes, por lo que no podemos tener un panorama próximo al original. Por último tenemos los "santuarios" con un número más restringido de zoomorfos, uno en Toro y Navarro, y dos en Victoria, complementados por diversos signos.

En el ámbito de la costa (Fig. 1), todas las cavidades con manifestaciones gráficas rupestres solutrenses aparecen asociadas a enclaves con ocupaciones de esta adscripción cronocultural (Nerja, Higuerón y Pileta) o muy cerca de los principales yacimientos solutrenses de la región (Toro de Bajondillo, mientras Navarro y Victoria se localizan muy próximos a los abrigos 4 y 6 de Humo e Higuerón). Este hecho creemos que pone de manifiesto un marcado carácter territorial, relacionado, a nuestro juicio, con una auténtica "apropiación simbólica" del contexto costero y de su ámbito de influencia inmediato (Simón y Cortés 2007) y estrechamente vinculado a la explotación de los territorios de subsistencia solutrenses de esta comarca.

En el interior (Fig. 1), Cueva de La Pileta dispone de un denso complejo topo-iconográfico estructurado en tres ciclos paleolíticos por H. Breuil (Breuil et al. 1915) y desglosado en el segmento solutrense en 5 horizontes (D-E-B-A-C) (Sanchidrián 1997). De éstos existe una datación directa obtenida de un bóvido (20.130 \pm 350$)$ del horizonte C (Sanchidrián y Valladas 2001). No obstante, la identificación de manos positivas y serpentiformes (Fortea 2005), considerando la antigüedad atribuida convencionalmente a estos motivos, inducen a pensar en que algunos de las representaciones agrupadas en los horizontes A y B puedan haber sido ejecutadas en momentos sincrónicos al desarrollo del Paleolítico superior inicial en la región que, como sabemos en la actualidad, arrancaría con el Auriñaciense y se consolidaría durante el Gravetiense (Cortés 2007, 2011, Aura et al. 2010). En este contexto y 
reconociendo que La Pileta constituye un complejo palimpsesto gráfico de dilatado recorrido cronológico, tenemos la percepción de que, con ser muy importante el ciclo Solutrense, la extensión temporal parece ser mucho mayor, en el sentido de los apuntado por J. Fortea (2005); aspecto sobre el que resultará en un futuro próximo necesario profundizar pues las propuestas más recientes de ordenación de los horizontes ha sido, a nuestro juicio, un poco errática. En la misma línea apunta también la secuencia arqueológica de La Pileta (Cortés y Simón 2008), que dispone de indicios relacionados quizás con la frecuentación de la cavidad durante el desarrollo de este último segmento crono-cultural.

Cueva de Doña Trinidad o Ardales por su parte cuenta también con un dispositivo topo-iconográfico de cronología muy amplia que cubre buena parte del Paleolítico superior y entre las que destaca significativamente el repertorio de representaciones zoomorfas solutrenses (Breuil 1921, Cantalejo et al. 2006), sobre todo caballos, algunos antropomorfos y signos grabados concentrados en una zona relativamente reducida de la cavidad.

Cueva de Nerja constituye el tercer yacimiento más rico en representaciones, en este caso básicamente pictóricas. Las asignadas al Solutrense muestran paralelos en otras cavidades andaluzas con las que comparte unas pautas compositivas similares. A nivel interno se han individualizado dos grandes conjuntos (Sanchidrián 1994). El ciclo más antiguo se localiza en las $G a$ lerías Altas de la cavidad y aparece caracterizado por figuras ubicadas en paneles bien despejados y de fácil visibilidad. Una datación por $\mathrm{C}^{14} / \mathrm{AMS}$ efectuada sobre un fragmento de carbón hallado en una pequeña cornisa situada a pocos centímetros por encima de una representación de cérvido dio una edad de $19.900 \pm 210$ B.P. (Sanchidrián y Valladas 2001) y encuadrada por tanto dentro del rango cronológico del Solutrense B de Nerja (Aura et al. 2006). También en las Galerías Altas de Nerja, se encuentran un grupo de figuras con características estilísticas adscribibles a momentos finales de la secuencia Solutrense, en concreto (Fig. 5) un conjunto de zoomorfos de la Sala de las Columnas de Hércules, dos équidos, una cierva y probablemente el denominado Camarín de los Peces (Cortés et al. en prensa). Además, todos ellos comparten entre otros atributos la ubicación en ámbitos espaciales de difícil visualización o la colocación de los animales en posición vertical (caballos Ne. 293 y Ne. 295) u oblicua (cérvido Ne. 294).

El segundo grupo de Nerja lo hallamos en las $G a-$ lerías Bajas y está caracterizado por una gran profusión de signos asociados a escasos zoomorfos, cierva como animal central asociada a un cáprido localizados en el área denominada Los Órganos. Esta misma composición se repite también en la Sala de la Cascada (Ne 35 a Ne 37). Desde el punto de vista cronoestilístico se encuadrarían en momentos avanzados del Solutrense, contemporáneos por tanto del Solutrense Tipo B de Aura y colaboradores (2006).

Por último, encontramos conjuntos finisolutrenses, encuadrados por edad en sentido amplio dentro del Solutrense C. Este sería el caso del denominado Panel del Puente, definido por el diseño de una cierva caracterizada por la prolongación del cuello y prótomos de dos caballos y un ciervo, con evidentes paralelismos en Ardales. A a estos momentos habría que sumar otros conjuntos del tramo final de la Sala del Cataclismo.

En cuanto al resto de cavidades, tendríamos que Toro, Navarro y Victoria contienen representaciones muy arcaicas en su concepción estilística y que quizás pudieran ser asignadas a momentos iniciales del Solutrense como apuntó ya Fortea (1978) o incluso podriamos retrotraernos a momentos presolutrenses en el caso de Toro y Victoria (Cortés et al. en prensa).

Frente a esta riqueza de manifestaciones rupestres, el arte mueble Solutrense es bastante pobre en la provincia de Málaga pues sólo aparece representado por algunas piezas en Nerja y Bajondillo (Sanchidrián 1994, Simón y Cortés 2007) y tan solo se ha podido identificar un ideomorfo.

En el apartado ornamental, a la espera del estudio de los elementos procedentes de Hoyo de la Mina, se limita al uso sistemático de moluscos marinos en los casos conocidos de Nerja y Tajo de Jorox y que ponen de manifiesto la presencia de una movilidad o red de intercambio de soportes malacológicos de origen marino desde la costa hasta el interior (Simón et al. 2006).

Para finalizar este apartado dedicado al ámbito simbólico cabe recordar la revisión del conjunto de enterramientos documentados en la Sala del Vestíbulo durante la campaña de 1962-1963. Aunque tradicionalmente han sido atribuidos al Solutrense, una datación directa mediante $\mathrm{C}^{14}$ /AMS del individuo Nerja-1 ha puesto de relieve que se trataba de una inhumación neolítica (Cortés et al. 2006). En este sentido, hay que reseñar que la datación fue obtenida a partir del análisis de un fragmento del húmero derecho de este individuo depositado en el Museo Arqueológico de Málaga. Este hecho conviene recordarlo, por cuanto el resto no fue afectado por el humo o el fuego, como ocurrió con el resto de la colección antropológica remitida en su día al Laboratorio de Antropología de la Universidad de Barcelona debido al incendio fortuito producido en esta institución, acontecido en 1969. 

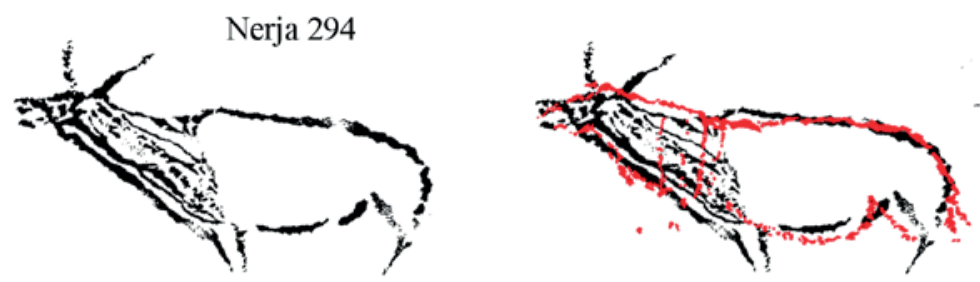

Nerja 295
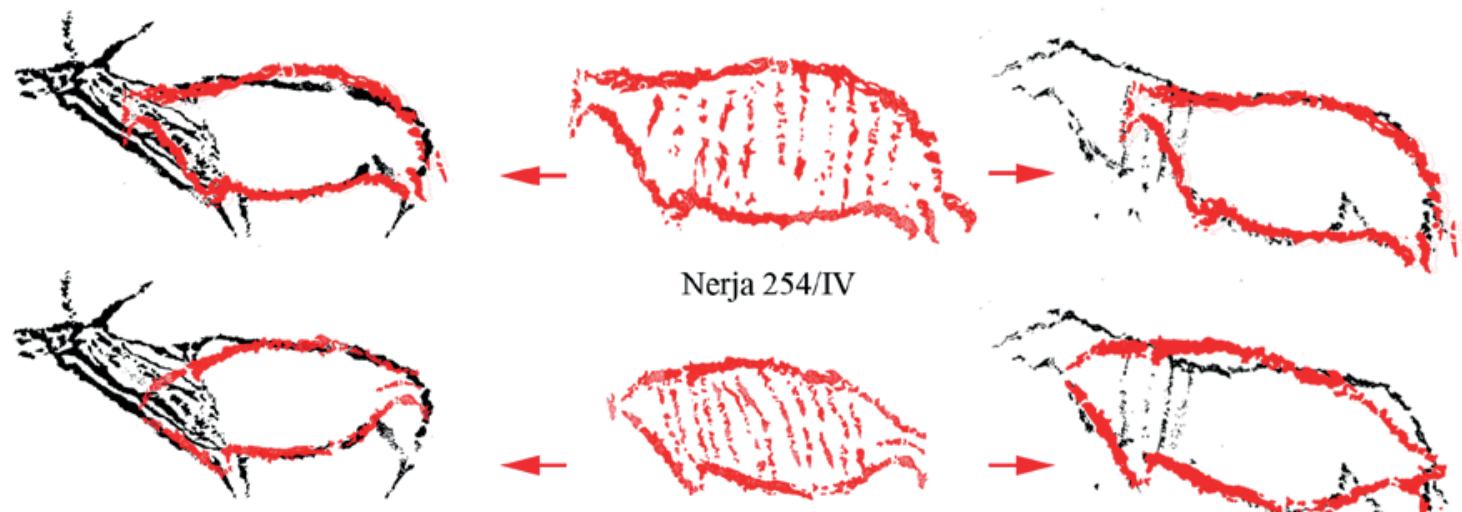

Nerja 254/IV
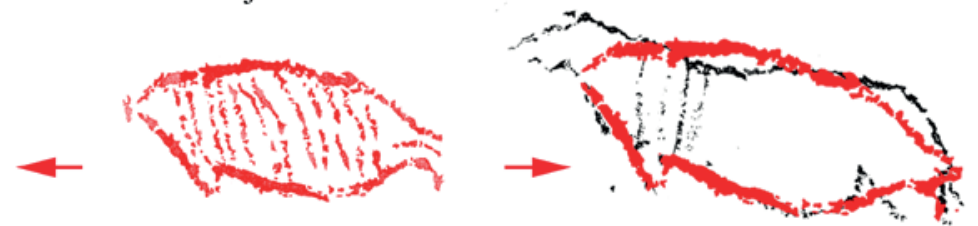

Nerja 254/III

Figura 5. Zoomorfos pintados del Solutrense evolucionado de la Sala de las Columnas de Hércules de la Cueva de Nerja (elaboración propia a partir de calcos de Sanchidrián 2004).

\section{DISCUSIÓN Y CONCLUSIONES}

El conocimiento del Solutrense en el extremo más meridional de la península Ibérica se sustenta básicamente en los yacimientos de la costa de Málaga. Esta distribución circunstancial, fruto más del azar de los hallazgos que heredera de una decidida investigación sobre esta área y temática, ha provocado un panorama aberrante en el que la costa polariza la mayor parte de los emplazamientos frente a unas cuantas estaciones con arte rupestre distribuidos en los macizos kársticos interiores.

En contraposición a esta distribución, los yacimientos del interior se circunscribían hasta hace poco a alguna colección descontextualizada (Tajo de Jorox) y a un conjunto de emplazamientos con un gran potencial investigador y que testimonian la circulación solutrense por el hinterland malagueño: Ardales o La Pileta (Cantalejo et al. 2006, Cortés et al. 2008). A este grupo hay que añadir una serie de sitios que, a buen seguro, enriquecerán en los próximos años el conocimiento que tenemos sobre el Solutrense meridional ibérico (Gorham, Higueral de Valleja, Hoyo de la Mina o La Pileta).
Desde el punto de vista estratigráfico, sedimentario y paleoambiental son interesantes las correlaciones que se pueden establecer entre el área objeto de nuestro estudio y el Levante ibérico, en concreto con la Cueva de Malladetes (Barx, Valencia), por poseer una adscripción cronocultural afín (Fumanal 1986, 1995). Así, podemos reseñar la coincidencia de procesos entre Nerja-Bajondillo y Malladetes:

Existencia de un hiatus cronoestratigráfico entre el Gravetiense y el Solutrense, documentado en Bajondillo y en Nerja. En Malladetes se detecta un horizonte de difícil adscripción cultural (N.VII) y de un "marcado empobrecimiento industrial”" (Fumanal 1986:77).

Durante el Solutrense inferior/pleno o tipo A y B de Aura y colaboradores (2006), se aprecia un ambiente frío o fresco. Esta segunda connotación se registra especialmente en Nerja, mientras que en Bajondillo es más árido en sus inicios pero, posteriormente, es más húmedo.

En Malladetes, el nivel VI, fechado con la máxima probabilidad (95\%) entre 27910-24270 años cal BP (Fig. 3) y adscrito al Solutrense inicial, se distingue por un medio húmedo con pulsaciones frías (Fumanal 1986), mientras que el nivel V, datado entre 25040 
y 23120 años cal BP con industrias del Solutrense pleno, se caracteriza por una humedad ambiental así como por el cese de las condiciones rigurosas (Fumanal 1986). Dicho horizonte cronocultural quedaría adscrito al Greenland stadial 3.

La transición cultural de Solutrense pleno a evolucionado se manifiesta estratigráficamente de forma distinta en los yacimientos de Málaga. Así, en Nerja se aprecia un contacto erosivo (entre NV 9 y NV 8), mientras que en Bajondillo es gradual (Bj/8). En Malladetes por su parte se detecta un hiatus cultural reflejado en el N IV del perfil Z-II, caracterizado por gelifractos (Fumanal 1986).

Durante el Solutrense evolucionado o tipo C de Nerja resulta complejo poder establecer una secuencia de episodios ya que, en Bajondillo hay algunas dataciones con una definición poco ajustada. A pesar de ello, podemos deducir que, en los registros analizados, se aprecia una tendencia hacia unas condiciones benignas y húmedas, más suaves que en el episodio anterior, correspondiente como indicamos al Solutrense inferior/ pleno. En Nerja, el NV8 se adscribiría al GS-2c, mientras que en Bajondillo y en Malladetes dicha secuencia parece ser más dilatada en el tiempo. En ella se aprecia una sucesión de distintos procesos que, en el caso de Bajondillo, se traducen en disgregaciones de las paredes, arroyadas, reactivaciones kársticas y erosiones que reflejan un ambiente más suave y húmedo, que podría correlacionarse al GS-2b; por su parte en el Levante correspondería a la Interfase D de Malladetes de M.P. Fumanal, cuyo techo estaría representado por el nivel III, fechado en $16.300 \pm 1.500 \mathrm{BP}$ y atribuido al Solutrense evolucionado o solutreogravetiense y caracterizado por “...unas condiciones ambientales templadas, biostáticas..." (Fumanal 1995: 120).

A nivel tecno-tipológico, los registros disponibles en la actualidad no son lo suficientemente diagnósticos como para articular una secuencia diacrónica clara y, en este sentido, tendríamos sólo las tendencias esbozadas en Nerja (Aura et al. 2006). Así, opinamos que los yacimientos de la costa de Málaga sólo permiten una agrupación genérica en dos segmentos (Cortés 2007), uno más antiguo, en el que están presentes los artefactos y esquemas operativos propios del Solutrense medio/ pleno, y otro más reciente o Solutrense evolucionado, en el que existen las características puntas de muesca o escotadura mediterránea.

El apartado funcional sólo ha podido analizarse de momento en una pequeña colección de Nerja, en la que sobresalen los instrumentos destinados al trabajo de materias animales como la carne, la piel y el hueso.
Por otra parte y desde el punto de vista del simbolismo, el Solutrense constituye el momento de mayor número de indicios de todo el Paleolítico superior meridional ibérico, al constatarse diversos "santuarios" integrados en los principales emplazamientos (Nerja e Higuerón), sus inmediaciones (Navarro y Victoria) o inscrito dentro de las presumibles áreas subsistenciales (Toro). No obstante, hay que reseñar que es posible que algunos de los "santuarios" puedan tener un origen que se remonte a momentos presolutrenses (p.ej. Toro, Navarro y Victoria). Así mismo, una aproximación tecnoestilística permite apuntar que el conjunto del " $\mathrm{Ca}$ marín de los Peces" de Nerja concuerda mejor con el conjunto gráfico más reciente del Solutrense (Cortés et al. en prensa).

El arte portátil es en contrapartida pobre y, por el momento, sin ninguna representación zoomorfa clara (Nerja y Bajondillo). Los adornos, presentes desde el Gravetiense en Nerja, se generalizan durante el Solutrense, fundamentalmente sobre moluscos marinos.

En resumen, aunque hay que reconocer la precariedad documental del Solutrense en el ámbito de Málaga, los datos disponibles ponen de manifiesto un poblamiento articulado por las áreas más próximas a la costa y apuntan a la existencia de un territorio subsistencial y simbólico plenamente adaptado a las condiciones paleoambientales rigurosas, especialmente durante sus inicios. Así mismo, lo datos sedimentoclimáticos obtenidos en los yacimientos de la costa de Málaga permiten su correlación con información similar procedente del Mediterráneo central ibérico y avanzar en la creación en un marco de referencia de escala macrorregional para el Solutrense.

\section{AGRADECIMIENTOS}

Este trabajo es una versión actualizada del trabajo presentado al Colloque international Le Solutréen 40 ans après la publication du Smith'66 celebrado en Preuilly-sur-Claise (Francia), 28-31 de octubre de 2007 y que nunca llegó a publicarse. Los resultados de este artículo han sido patrocinados por la Fundação para a Ciência e a Tecnologia (Portugal) and the European Science Foundation (III Community Support Framework) y se han obtenido en el marco del proyecto "Estudio y contextualización de las antiguas excavaciones del Patronato de la Cueva de Nerja. 1959-1978" autorizado por la Consejería de Cultura de la Junta de Andalucía. 


\section{BIBLIOGRAFÍA}

ANDERSEN, K.K.; SVENSSON, A.; JOHNSEN, S.J.; RASMUSSEN, S.O.; BIGLER, M.; THLISBERGER, R.; RUTH, U.: SIGGARD-ANDERSEN, M-L.; STEFFENSEN, J.P.; DAHL-JENSEN, D.; VINTHER, B.M. y CLAUSEN, H.B. (2006): "The Greenland Ice Core Chronology 2005, 1542 ka. Part 1", Constructing the timescale. Quaternary Science Reviews 25: 3246-3257. http://dx.doi. org/10.1016/j.quascirev.2006.08.002

AURA, J.E.; JORDÁ, J.F.; PÉREZ, M. y RODRIGO, M.J. (2001): "Sobre dunas, playas y calas. Los pescadores prehistóricos de la Cueva de Nerja (Málaga) y su expresión arqueológica en el tránsito Pleistoceno-Holoceno", Archivo de Prehistoria Levantina 35: 9-39.

AURA, J.E.; JORDÁ, J.F. y FORTEA, F.J. (2006): “La cueva de Nerja (Málaga, España) y los inicios del Solutrense en Andalucía", Zephyrus 59: 67-88.

AURA, J.E.; JORDÁ, J.F.; PÉREZ, M.; BADAL, E.; MORALES, J.V.; AVEZUELA, B.; TIFFAGOM, M. y JARDÓN, P. (2010): "Treinta años de investigación sobre el Paleolítico superior de Andalucía: la Cueva de Nerja”, en X. Mangado (ed.), El Paleolítico superior peninsular. Novedades del siglo XXI. Homenaje al profesor Javier Fortea. Monografies SERP 8: 173-198. Universidad de Barcelona, Barcelona.

AURA, J.E.; JORDÁ, J.F.; PÉREZ, M. y RODRIGO, M.J. (2002): "The Far South: The Pleistocene-Holocene transition in Nerja Cave (Andalucia, Spain)", Quaternary International 93-94: 19-30.

BARROSO, C.; MEDINA, F.; ONORATINI, G. y JÖRIS, C. (2006): "Les industries du Paléolithique Supérieur (Protoaurignacien, Gravettien et Solutréen) de la grotte du Boquete de Zafarraya", en C. Barroso y H. de Lumley (ed.), La grotte du Boquete de Zafarraya. Málaga. Andalousie: $1587-$ 1626. Junta de Andalucía.

BERGADÀ, M.M. y CORTÉS, M. (2007): "Secuencia estratigráfica y sedimentaria”, en M. Cortés (ed.), Cueva de Bajondillo (Torremolinos). Secuencia cronocultural y paleoambiental del Cuaternario reciente en la Bahía de Málaga: 93-138. Servicio de publicaciones, Centro de publicaciones de la Diputación de Málaga, Málaga.

BREUIL, H. (1921): «Nouvelles cavernes ornées paléolithique dans la province de Málaga», L'Anthropologie XXXI: 239-250.

BREUIL, H.; OBERMAIER, H. y VERNER, W. (1915): La Pileta à Benaoján (Málaga) (Espagne). Institute de Paléontologie Humaine, Mónaco.
CACHO, I.; GRIMALT, J.O.; CANALS, M.; SBAFFI, L.; SHACKLETON, N.J.; SCHÖNFELD, J.yZAHN, R. (2001): "Variability of the western Mediterranean Sea surface temperature during the last 25,000 years and its connection with the Northern Hemisphere climatic changes", Paleoceanography 16/1: 40-52. http://dx.doi.org/10.1029/2000PA000502

CACHO, C. y LÓPEZ, P. (1979): "La Cueva del Higuerón (Málaga). Estudio de sus materiales", Trabajos de Prehistoria 36/1: 11-82.

CANTALEJO, P.; MAURA, R.; ARANDA, A. y ESPEJO, M.M. (2007): Prehistoria en las cuevas del Cantal. Editorial La Serranía, Málaga.

CANTALEJO, P.; MAURA, R.; ARANDA, A.; ESPEJO, M.M.; RAMOS, F.; MEDIANERO, J.; ARANDA, A. y DURÁN, J.J. (2006): La Cueva de Ardales: Arte prehistórico y ocupación en el Paleolítico Superior. Centro de publicaciones de la Diputación de Málaga, Málaga.

CORTÉS, M. (ed.) (2007a): Cueva de Bajondillo (Torremolinos). Secuencia cronocultural y paleoambiental del Cuaternario reciente en la Bahía de Málaga. Servicio de publicaciones, Centro de publicaciones de la Diputación de Málaga, Málaga.

- (2007b): El Paleolítico Medio y Superior en el sector central de Andalucía (Córdoba y Málaga). Monografías Museo de Altamira 22. Ministerio de Cultura, Madrid.

- (2010): "El Paleolítico superior en el sur de la Península Ibérica. Un punto de partida a comienzos del siglo XXI", en X. Mangado (ed.), El Paleolítico superior peninsular. Novedades del siglo XXI. Homenaje al profesor Javier Fortea. Monografies SERP 8: 173-198. Universidad de Barcelona, Barcelona.

CORTÉS, M.; BERGADÀ, M.M.; GIBAJA, J.F., JIMÉNEZ, F.; SIMÓN. M.D. y RIQUELME, J.A. (2011): "El Solutrense en la costa de Málaga: contexto paleoambiental y cronocultural", Pyrenae 421: 51-75.

CORTÉS, M.; BICHO, N.F.; MENDONÇA, C. y SIMÓN, M.D. (en prensa): "In fine Iberia terram: El final del Paleolítico superior en el extremo occidental de Europa", Homenaje al profesor Javier Fortea. Universidad de Oviedo, Oviedo.

CORTÉS, M.; JIMÉNEZ, F.J.; SIMÓN, M.D.; GIBAJA, J.F.; CARVALHO, A.F.; MARTÍNEZRUIZ, F.; RODRIGO, M.; FLORES, J.A.; PAYTAN, A.; LÓPEZ, J.A.; PEÑA-CHOCARRO, L.; CARRIÓN, J.S.; MORALES, A.; ROSELLÓ, E.; RIQUELME, J.A.; DEAN, R.M.; SALGUEIRO, 
E.; MARTÍNEZ, R.M.; DE LA RUBIA, J.J.; LOZANO, M.C.; VERA, J.L. y BICHO, N.F. (2012): "The Mesolithic-Neolithic transition in southern Iberia", Quaternary Research 77/2: 221-234. http:// dx.doi.org/10.1016/j.yqres.2011.12.003

CORTÉS, M.; MORALES, A.; SIMÓN, M.D.; BERGADÀ, M.M.; DELGADO, A.; LÓPEZ, P., LÓPEZ, J.A., LOZANO, M.C., RIQUELME, J.A., ROSELlÓ, E., SÁNCHEZ, A. y VERA, J.L. (2008): "Paleoenvironmental and cultural dynamics of the Coast of Malaga (Andalusia, Spain) during the Upper Pleistocene and Early Holocene", Quaternary Science Reviews 27: 176-219. http://dx.doi. org/10.1016/j.quascirev.2008.03.010

CORTÉS, M. y SANCHIDRIÁN, J.L. (1999): “Dinámica cultural del Pleistoceno Superior en la costa de Málaga”, Cuaternario y Geomorfología 13: 63-77.

CORTÉS, M. y SIMÓN, M.D. (1997): “Cueva Bajondillo (Torremolinos, Málaga). Aportaciones al Paleolítico en Andalucía", en El món Mediterrani després del Pleniglacial (18.000-12.000 BP): 275-290. Centro de Investigaciones Arqueológicas de Gerona, Gerona.

- (2007): "La Pileta (Benaoján, Málaga) cien años después. Aportaciones al conocimiento de su secuencia arqueológica", Saguntum 40: 45-64.

CORTÉS, M.; SIMÓN, M.D.; FERNÁNDEZ, E.; GUTIÉRREZ, C.; MORALES, A.; LOZANO, M.C.; ROSELLÓ, E.; RIQUELME, J.A.; TURBÓN, D. y VERA, J.L. (2006): "Nuevos datos sobre el Paleolítico superior en la Cueva de Nerja (Andalucía, España)", en J.L. Sanchidrián, A.M. Márquez y J.M. Fullola (eds.), Reunión de la Cuenca mediterránea durante el Paleolítico Superior (38.000-10.000): 298-314. Fundación Cueva de Nerja, Málaga.

CORTÉS, M.; SIMÓN, M.D.; RIQUELME, J.A.; PEÑA, L; GIBAJA, J.F.; DE LA RUBIA, J.J. y MARTÍNEZ, R.M. (2010): “El Neolítico en la costa de Málaga (España): viejos y nuevos datos para su contextualización en el proceso de neolitización del sur de la península Ibérica" en J.F. Gibaja y A. Faustino (eds.), Os últimos caçadores-recolectores e as primeiras comunidades productoras do sul da Península Ibérica e do norte de Marrocos. Promontoria Monográfica 15: 151-162.

CORTÉS, M.; SIMÓN, M.D.; MARREIROS, J.; GIBAJA, J. y BICHO, N.F. (en prensa): "Reevaluación del Gravetiense en el sur de Iberia. Coloquio Gravetiense Cantábrico: estado de la cuestión", Museo de Altamira, 20-22 de octubre de 2011. En preparación.
FERRER, J.E; MARQUÉS, I.; CORTÉS, M.; BALDOMERO, A. y RAMOS, J. (2006): “Excavaciones en Cueva del Hoyo de la Mina (Málaga, Andalucía, España). Contrastación de una secuencia arqueológica clásica para el estudio del Tardiglaciar-Holoceno antiguo en el sur de la Península Ibérica", en J.L. Sanchidrián, A.M. Márquez y J.M. Fullola (eds.), Reunión de la Cuenca mediterránea durante el Paleolítico Superior (38.000-10.000): 316-325. Fundación Cueva de Nerja, Málaga.

FORTEA, F.J. (1973): Los complejos microlaminares y geométricos del Epipaleolítico Mediterráneo Español. Memorias del Seminario de Prehistoria y Arqueología 4. Universidad de Salamanca, Salamanca.

- (2005): "La plus ancienne production artistique du Paléolithique ibérique", en Actas del Simposio Pitture paleolitiche nelle Prealpi venete: Grotta di Fumane e Riparo Dalmieri: 89-99. Museo Civico di Storia Naturale di Verona, Verona.

FORTEA, F.J. y GIMÉNEZ, M. (1973): “La Cueva del Toro. Nueva estación malagueña con Arte Paleolítico", Zephyrus XX: 6-16.

FUMANAL, M.P. (1986): Sedimentología y clima en el país valenciano. Las cuevas habitadas en el cuaternario reciente. Servicio de Investigación Prehistórica Serie de Trabajos Varios 83. Diputación Provincial de Valencia, Valencia.

FUMANAL, M.P. (1995): "Los depósitos cuaternarios en cuevas y abrigos. Implicaciones sedimentoclimáticas", El Cuaternario del País Valenciano: 115124. Universidad de Valencia, Valencia.

JORDÁ, J.F. (1986): La prehistoria de la Cueva de Nerja. Trabajos sobre la Cueva de Nerja 1: 145172. Patronato de la Cueva de Nerja. Málaga.

JORDÁ, J.F. y AURA, J.E. (2006): "Radiocarbono, cronoestratigrafía y episodios ocupacionales en el Pleistoceno superior y Holoceno de la cueva de Nerja (Málaga, Andalucía, España)", en J.M. Maí1 lo y J.M. Baquedano (ed.), Miscelánea en homenaje a Victoria Cabrera. Zona Arqueológica 7: 578-597.

LOWE, J.J., RASMUSSEN, S.O., BJÖRCK, S., HORK, W.Z., STEFFENSEN, J.P., WALKER, M.J.C. y YU, Z.C. (2008): "Synchronisation of palaeoenvironmental events in the North Atlantic region during the Last Termination: a revised protocol recommended by the INTIMATE group", Quaternary Science Reviews 27: 6-17. http://dx.doi. org/10.1016/j.quascirev.2007.09.016

MARQUÉS, I. y RUIZ, A.C. (1976): "El Solutrense de la Cueva del Tajo de Jorox, Alozaina (Málaga)", 
Cuadernos de Prehistoria de la Universidad de Granada 1: 47-58.

RAMOS, J.; CORTÉS, M.; AGUILERA, A.; LOZANO, M.C.; VERA, J.L. y SIMÓN, M.D. (2006): "El Magdaleniense y Epipaleolítico del Abrigo 6 del Complejo del Humo (La Araña, Málaga)", en J.L. Sanchidrián, A.M. Márquez y J.M. Fullola (eds.), Reunión de la Cuenca mediterránea durante el Paleolítico Superior (38.000-10.000): 326-341. Fundación Cueva de Nerja, Málaga.

RASMUSSEN, S.O., ANDERSEN, K.K., SVENSSON, A.M., STEFFENSEN, J.P.; VINTHER B.M.; CLAUSEN, H.B.; SIGGAARD-ANDERSEN, M.L.; JOHNSEN, S.J.; LARSEN, L.B.; DAHLJENSEN, D.; BIGLER, M.; RÖTHLISBERGER, R.; FISCHER, H.; GOTO-AZUMA, K.; HANSSON, M.E. y RUTH, U. (2006): “A new Greenland ice core chronology for the last glacial termination", Journal of Geophysical Research 111, D06102. http://dx.doi.org/10.1029/2005JD006079.

RIQUELME, J.A.; SIMÓN, M.D. y CORTÉS, M. (2005): "La fauna de mamíferos del Solutrense en la Cueva de Nerja. La fauna de mamíferos del Solutrense en la Cueva de Nerja", Munibe 57/1: 255263.

SANCHIDRIÁN, J.L. (1981): Cueva Navarro (Cala del Moral, Málaga). Corpus Artis Rupestris. I Palaeolithica Ars. Vol. 1. Universidad de Salamanca, Salamanca.

- (1994): Arte Rupestre de la Cueva de Nerja. Trabajos sobre la Cueva de Nerja 4. Patronato de la Cueva de Nerja, Málaga.

- (1997): "Propuesta de la secuencia figurativa en la Cueva de La Pileta", Centre d'Investigacions Arqueològiques, Girona. Sèrie Monogràfica 17. El món mediterrani després del Pleniglacial (18.00012.000 B.P.): 411-430. Centro de Investigaciones Arqueológicas de Gerona, Gerona.

SANCHIDRIÁN, J.L. y VALLADAS, H. (2001): “Dataciones numéricas del arte rupestre de la cueva de La Pileta (Málaga, Andalucía)", Panel 1: 104-105.

SIMÓN, M.D. (2003): "Una secuencia con mucha prehistoria: la Cueva de Nerja", Mainake XXV: 49-274.

SIMÓN, M.D. y CORTÉS, M. (2007): "Arte mueble de Cueva Bajondillo en el contexto de las manifestaciones artísticas pleistocenas de la Bahía de
Málaga”, en M. Cortés (ed. y coord.), Cueva Bajondillo (Torremolinos). Secuencia cronocultural y paleoambiental del Cuaternario reciente en la Bahía de Málaga: 447-452. Servicio de Publicaciones del Centro de Ediciones de la Diputación de Málaga, Málaga.

SIMÓN, M.D.; NAVARRETE, I.; CORTÉS, M.; LOZANO, M.C. y VERA, J.L. (2006): "Nuevos elementos simbólicos sobre soporte malacológico del Paleolítico Superior de la provincia de Málaga (Andalucía, España)", en J.L. Sanchidrián, A.M. Márquez y J.M. Fullola (eds.), Reunión de la Cuenca mediterránea durante el Paleolítico Superior (38.000-10.000): 366-378. Fundación Cueva de Nerja, Málaga.

SUCH, M. (1920): Avance al estudio de la caverna de "Hoyo de la Mina” en Málaga, Boletín de la Sociedad Malagueña de Ciencias, Málaga.

SVENSSON, A.; ANDERSEN, K.K.; BIGLER, M.; CLAUSEN, H.B.; DAHL-JENSEN, D.; DAVIES, S.W.; JOHNSEN, S.J.; MUSCHELER, R.; RASMUSSEN, S.O.; RÖTHLISBERGER, R.; STEFFENSEN, J.P. y VINTHER, B.M. (2006): “The Greenland Ice Core Chronology 2005, 15-42 ka. Part 2: comparison to other records", Quaternary Science Reviews 25: 3258-3267. http://dx.doi. org/10.1016/j.quascirev.2006.08.003

VINTHER, B.M.; CLAUSEN, H.B.; JOHNSEN, S.J.; RASMUSSEN, S.O.; ANDERSEN, K.K.; BUCHARDT, S.L.; DAHL-JENSEN, D.; SEIERSTAD, I.K.; SIGGAARD- ANDERSEN, M.-L.; STEFFENSEN, J.P.; SVENSSON, A.; OLSEN, J. y HEINEMEIER, J. (2006): "A synchronized dating of three Greenland ice cores throughout the Holocene", Journal of Geophysical Research 111, D13102. Doi: 10.1029/2005JD006921

WENINGER, B. y JÖRIS, O. (2004): “Glacial Radiocarbon Calibration. The CalPal Program. Radiocarbon and Archaeology", en T. Higham, C.B. Ramsey y C. Owen (eds.), Fourth International Symposium, Oxford.

WENINGER, B.; JÖRIS, O. y DANZEGLOCKE, U. (2007): "CalPal-University of Cologne Radiocarbon Calibration Program Package CalPal2007_ HULU', Institut der Ur-und Frühgeschite, Universität zu Köln. Köln (http://www.calpal.de).

FECHA DE ENTRADA: 26/01/2012

FECHA DE ACEPTACIÓN: 27/02/2012 